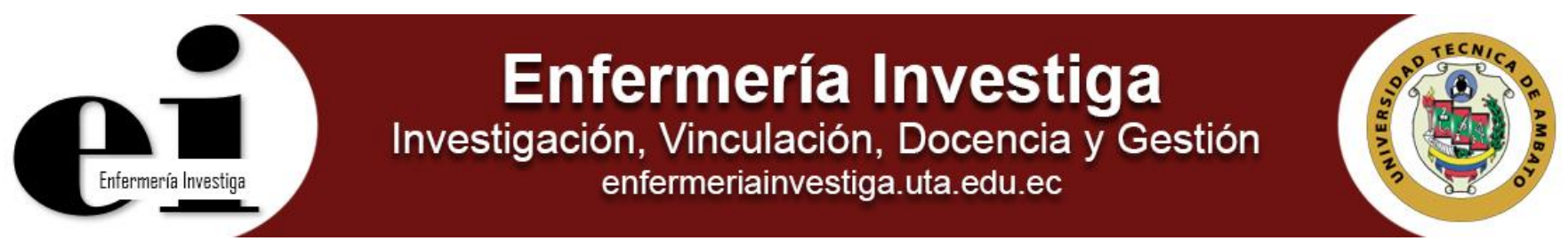

DOI: http://dx.doi.org/10.29033/ei.v2n4.2017.06

Artículo original

\title{
Lecciones aprendidas de dengue y chikungunya
}

Lessons learned from dengue and chikungunya

Janette Ester Eras Carranza1, Imelda Gumercinda Arias Montero², Miriam Ivonne Fernández Nieto², Lilian Marisol Floreano Solano ${ }^{1}$, Sara Margarita Saraguro Salinas ${ }^{1}$, Jovanny Angelina Santos Luna ${ }^{1}$, Ana Marina Vaca Gallegos ${ }^{1}$

\footnotetext{
${ }^{1}$ Universidad Técnica de Machala - Facultad Ciencias Químicas y de la Salud - Carrera de Enfermería - Machala - Ecuador

2 Universidad Estatal de Milagro - Facultad Ciencias de la Salud - Carrera de Enfermería - Milagro - Ecuador

${ }^{3}$ Universidad Técnica de Ambato - Facultad Ciencias de la Salud - Carrera de Enfermería - Ambato - Ecuador
}

Eras CJE, Arias MIG, Fernández NMI, Floreano SLM, Saraguro SSM, Santos LJA, Vaca GAM. Lecciones aprendidas de dengue y chikungunya. Enferm Inv (Ambato). 2017; 2(4): 150-155.

2477-9172 / 2550-6692 Derechos Reservados ( 2017 Universidad Técnica de Ambato, Carrera de Enfermería. Este es un artículo de acceso abierto distribuido bajo los términos de la Licencia Creative Commons, que permite uso ilimitado, distribución y reproducción en cualquier medio, siempre que la obra original es debidamente citada.

\section{Historia:}

Recibido: 30 agosto 2017

Revisado: 02 noviembre 2017

Aceptado: 04 diciembre 2017

Palabras Claves: Dengue; chikungunya; virosis; epidemiología descriptiva

Keywords: Dengue; chikungunya; virus diseases: descriptive epidemiology

\section{Resumen}

Introducción: El dengue y chikungunya son infecciones víricas transmitidas por mosquitos. El cuadro clínico varía de simple hasta grave, la carga de enfermedad está invisibilizada en la comunidad al no acceder a los servicios de salud por características atípicas.

Objetivo: Describir las características del dengue y Chikungunya en la ciudad de Machala en el año 2015.

Métodos: Se realizó estudio descriptivo de la situación epidemiológica de dengue y chikungunya por medio de la construcción del canal endémico con tasas de incidencia semanal de ocho años.

Resultados: En el año 2015 fueron 15 semanas de epidemia que coincidió con las semanas de la estación invernal (enero a mayo); el chikungunya apareció en la semana 21 . El grupo de edades más afectado fue el de 20 a 49 años del sexo femenino con $55.7 \%$. Entre las medidas implementadas de prevención y control del dengue y chikungunya, fue la fumigación en localidades de riesgo, visitas domiciliarias y además se trabajó con larvicida obteniéndose un índice de depósitos positivos de $1.47 \%$.

Conclusiones: El dengue fue endémico y los casos se exacerbaron en estación invernal. Coincidieron como factores de riesgo la presencia del mismo vector transmisor, población expuesta al vector por las prácticas culturales y clima tropical. La diferencia entre dengue y chikungunya estuvo en los cuatros serotipos para dengue y uno para chikungunya, determinando el riesgo y la tendencia mayor para el dengue.

\section{Abstract}

Introduction: Dengue and chikungunya are viral infections transmitted by mosquitoes. The clinical picture varies from simple to severe, the burden of disease is made invisible in the community by not accessing health services due to atypical characteristics.

Objetive: Describe the characteristics of dengue and Chikungunya in the city of Machala in 2015

Methods: A descriptive study of the epidemiological situation of dengue and chikungunya was carried out through the construction of the endemic channel with weekly incidence rates of eight years.

Results:

In 2015 there were 15 weeks of epidemic that coincided with the weeks of the winter season (January to May); chikungunya appeared in week 21 . The most affected age group was women 20 to 49 years old with $55.7 \%$. Among the implemented measures for the prevention and control of dengue and chikungunya, was the spraying in localities at risk, home visits and also worked with larvicide obtaining an index of positive deposits of $1.47 \%$.

Conclusions: Dengue fever was endemic and cases were exacerbated in the winter season. The presence of the same transmitting vector, population exposed to the vector by cultural practices and tropical climate, coincided as risk factors. The difference between dengue and chikungunya was in the four serotypes for dengue and one for chikungunya, determining the risk and the greater tendency for dengue.

Autor de correspondencia:

Janette Ester Eras Carranza. Carrera de Enfermería, Universidad Técnica de Machala, Km.5 1/2 Vía Machala Pasaje, Teléfono: +593 072983362 , Machala, Ecuador. E-mail: jeras@utmachala.edu.ec 


\section{Introducción}

El dengue y el chikungunya son infecciones víricas transmitidas por mosquitos, debido a cuatro serotipos. El cuadro clínico es variante y va desde un cuadro clínico simple hasta uno grave, caracterizado por síntomas gripales y en ocasiones evoluciona hasta convertirse en un cuadro potencialmente mortal llamado dengue grave. Por su parte el chikungunya se presenta como un cuadro clínico agudo, con secuelas de artritis que duran de dos meses a dos años. Las estimaciones recientes utilizando enfoques cartográficos sugieren que este número se acerca a casi 400 millones. Se espera que la expansión del dengue aumente debido a factores tales como la dinámica moderna del cambio climático, la globalización, los viajes, el comercio y la evolución viral ${ }^{1}$.

La problemática de las enfermedades vectoriales como dengue y chikungunya, mediante el uso de aproximaciones cartográficas, estiman unos 390 millones de infecciones de dengue por año, de las cuales 96 millones se manifiestan aparentemente. El total de infecciones es más de tres veces la estimación de la carga de dengue según la Organización Mundial de la Salud (OMS) ${ }^{2}$. Esta situación alerta a nivel local por la magnitud del problema y la carga de enfermedad invisible en la comunidad al no acceder a los servicios de salud. Un estudio en una escuela de Bangkok arrojó que el $87 \%$ de escolares se infectaron con el virus del dengue y el $53 \%$ de casos fueron identificados como dengue hemorrágico requiriendo hospitalización ${ }^{3}$. De hecho, la presencia primaria de dengue deja sensibilizada a la persona para que se produzca un dengue grave con la segunda infección, por lo que es importante conocer el nivel de sensibilidad que tiene la población y estar preparado en los servicios de salud. Con respecto a las enfermedades vectoriales América Latina, está el reto de enfrentar enfermedades emergentes como es chikungunya, sumando a la carga de morbilidad del dengue. Kuorí (2006), estableció que más de 2500 millones de personas viven en zonas en riesgo de dengue ${ }^{4}$.

Existen estudios que demuestran la dinámica de la patogenicidad de la enfermedad, cuando la presencia de anticuerpos neutraliza la infección al unirse a partículas del virus en cantidades suficientemente grandes. Por el contrario, en concentraciones que caen por debajo del umbral de neutralización, los anticuerpos pueden promover la entrada de virus del dengue en células que expresan receptores ${ }^{5}$. Robinson y colaboradores, reportaron que el anticuerpo Ab513 mitiga la trombocitopenia en un modelo de ratón humanizado, resuelve la fuga vascular, reduce la viremia a niveles casi indetectables y protege a los ratones, demostrando así que el anticuerpo Ab513 puede reducir la carga de salud pública del dengue ${ }^{6}$. Es importante destacar que un inhibidor alostérico Hsp70, JG40, bloquea potencialmente la infección de diferentes serotipos de dengue en células sanguíneas primarias humanas sin provocar resistencia viral o ejercer toxicidad en las células huésped ${ }^{7}$. El conocimiento del virus es importante debido a que se puede inferir el impacto en la prevalencia en la comunidad para la toma de decisiones en relación a la carga de la enfermedad con sus niveles de gravedad.

Entre los factores de riesgo, están los estilos de vida que tiene la población con la exposición en las horas de picada que es en el día, además de cultura barroca de la reutilización de objetos inservibles que se acumulan en los patios de las viviendas convirtiéndose en depósitos de riesgo para la formación de criaderos de larvas de mosquito Aedes aegypti. En el contexto ambiental se tiene como problemas que coadyuvan en la presencia de criaderos de larvas de mosquito Aedes, la acumulación de agua para el consumo humano que en muchos casos se encuentra al descubierto, siendo accesible para que el mosquito hembra deposite sus huevos.

Los países de Latinoamérica tienen ahora hiperendemicidad con epidemias de dengue y transmisión que ocurre cíclicamente en la región cada tres a cinco años, con frecuencia cada vez mayor ${ }^{1}$. Esta situación da como resultado una mayor sensibilidad de las personas al presentar ya un episodio de dengue, estando en manos del personal de salud la solución con la atención de salud en base a signos de alarma y casos graves que se presentan. Burke en el año 1998 indicó que, la situación para los países de Latinoamérica, incluyendo Ecuador, se torna en preocupante cuando se introduce el nuevo virus de chikungunya, a una población susceptible.

El estudio de McBride plantea que el mosquito Aedes aegypti en la forma doméstica coexiste con una forma ancestral de "bosque" que prefiere morder animales no humanos y se encuentra a lo largo de la costa de Kenia. Los mosquitos domésticos han evolucionado con una marcada preferencia por el olor corporal humano. Las colonias de preferencia humana procedentes de Kenia, Tailandia, Estados Unidos y África Occidental estaban dominadas por alelos Alike, mientras que las colonias de preferencia animal de Kenya y Uganda eran todas muy variables ${ }^{8}$; además que el extracto de glándula salival (SGE) directamente perturba la función de la barrera endotelial in vitro e induce la permeabilidad endotelial in vivo en la piel. EI SGE aumenta los títulos virales en la piel, aumenta la infección por virus del dengue de células dendríticas y macrófagos en la dermis, y amplifica la migración de células dendríticas a los ganglios linfáticos que drenan la piel ${ }^{9}$.

Para la transmisión del dengue y chikungunya es necesaria la presencia del Aedes aegypti, por lo cual se manejan indicadores como son: índice de casa positiva, índice de depósitos e índice de Breteau, indicador este que relaciona el riesgo de transmisión ${ }^{10}$, pues sobre cinco criaderos positivos por 100 casas inspeccionadas tienen un riesgo alto para la transmisión de virus de dengue, al aumentar de esta manera la densidad vectorial. Una situación que hay que considerar son los cambios que se producen en la naturaleza que originan la rápida adaptación de patógenos a estos cambios ecológicos, dada directamente como consecuencia de las actividades humanas ${ }^{11}$.

El objetivo del presente artículo es de describir las características de dengue y chikungunya en la ciudad de Machala en el año 2015. Para lo cual se debe caracterizar ambas enfermedades por espacio, tiempo y persona; determinar las medidas implementadas para la prevención y control y medir la tendencia del dengue.

\section{Materiales y métodos}

Se realizó un estudio observacional descriptivo de la situación epidemiológica de dengue y chikungunya en el Distrito Machala, provincia el Oro, Ecuador, para la construcción del canal endémico. Fueron calculadas las tasas de incidencia semanal de ocho años de observación y la transformación logarítmica de la misma, para calcular la media semanalmente, desviación estándar y realizar la transformación a unidades originales de tasas a casos esperados en números absolutos.

La curva epidémica se graficó según la frecuencia semanal de los casos que se presentaron en el año 2015, en comparación con los casos presentados en el año 2014. La 
caracterización por persona estuvo en función de las variables de edad y sexo de los casos presentados.

Los indicadores vectoriales, estuvieron relacionados con índice de casa positiva, que fueron las casas en las que se encontró criaderos de Aedes aegypti positivos en relación con el total de casas inspeccionadas por 100. El índice de depósito se estableció según los depósitos encontrados como positivos en relación con el total de depósitos inspeccionados por 100. El índice Breteau se determinó como los depósitos positivos relacionados con el total de casas inspeccionadas por $100^{12,13}$.

\section{Resultados}

Luego de la sistematización realizada se pudo apreciar los instrumentos de monitoreo de dengue y chikungunya, se exponen los casos presentados de dengue del año 2015, según las variables de tiempo, lugar y persona.

Al observar la curva de los dos años de comparación (figura 1 ), tiene un inicio muy discreto con aumento de los casos haciendo el pico mayor en la semana 24 , descendiendo de forma paulatina, evidenciando una epidemia propagada. En la comparación de los años 2014 y 2015, se apreció una diferencia de 1591 casos, siendo 2.3 casos presentados en el año 2015 a un caso presentado en el año 2014. En el periodo de aumento de casos (semana 19 a semana 33) en el año 2014 concentró el $78.3 \%$, mientras que en el año 2015 concentró el 89.3\%, existiendo una duración de 15 semanas de aumento de casos en relación al año 2014.

Figura 1. Curva epidémica comparativa de dengue entre los años 2014 - 2015, Machala.

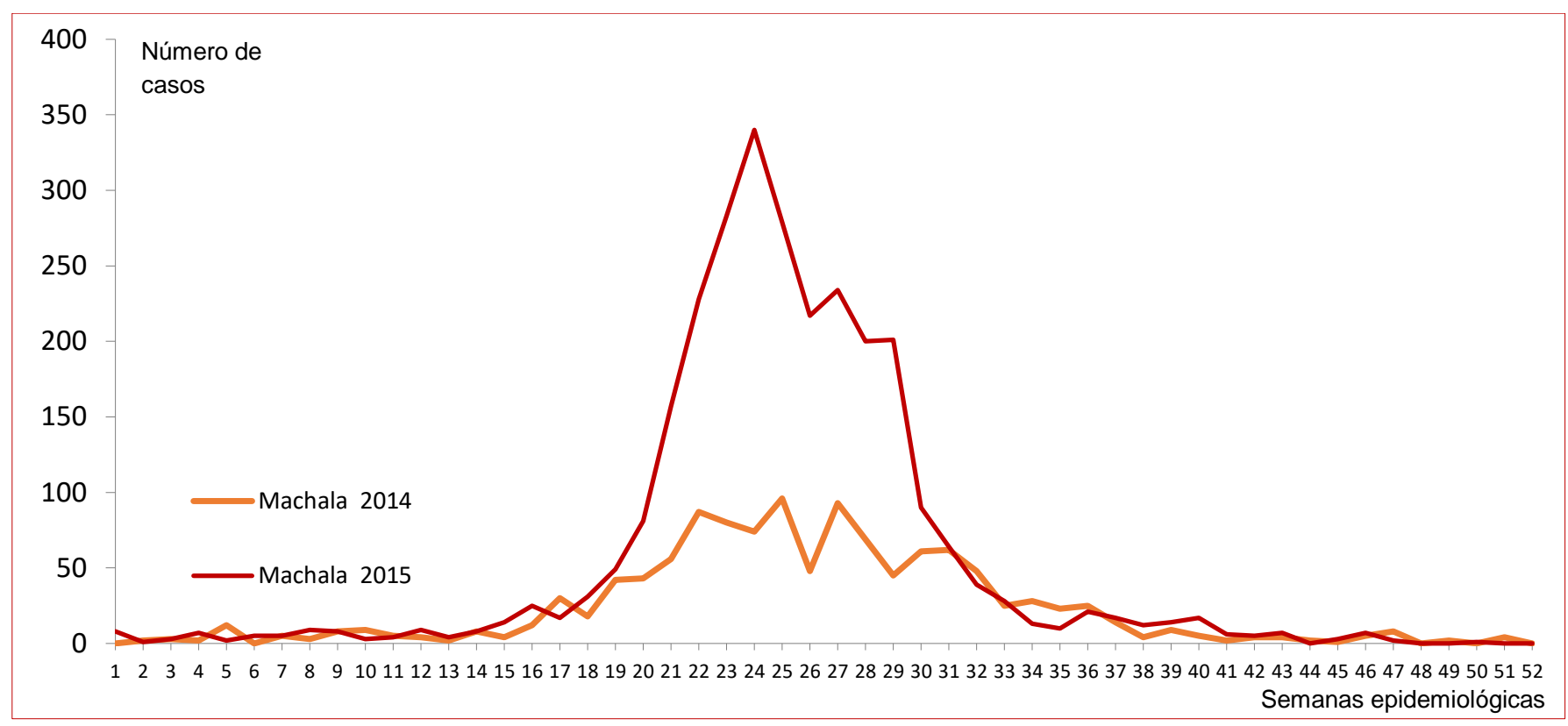

Fuente: Notificación del Sistema de Vigilancia Epidemiológica.

La presencia de dengue en el monitoreo del canal endémico en las primeras semanas epidemiológicas (semana 1 a 17) se encontró dentro del canal en el rango de seguridad, sin embargo, en la semana 18 hubo un aumento incipiente y ya en la semana 19 y 20 fue más notorio el aumento de casos, ingresando a nivel de epidemia y se mantuvo hasta la semana 33 (3 de mayo al 15 de agosto 2015), para a partir de la semana 34 ingresar a nivel de seguridad. Se observó 15 semanas en epidemia siendo coincidente con la etapa invernal (figura 2).

La tabla 1 representa la caracterización de edad y sexo de los casos de dengue presentados en la ciudad de Machala. El sexo más afectado fue el femenino con el 55.7\%, mientras que el grupo poblacional que presentó más casos fue el de 20 a 49 años de edad con $44.7 \%$; la diferencia entre sexos no fue significativa.
De hecho, el aumento en el número de casos de dengue rebasa el intervalo de confianza superior a la fecha de la estación invernal (enero a mayo), sin embargo, hay que recordar que el mosquito Aedes aegypti transmisor del dengue, se encuentra conviviendo con las personas dentro de la casa, y por lo tanto el nicho ecológico es el adecuado sobre todo cuando encuentra depósitos de agua para depositar los huevos.

La curva epidémica (figura 3), representa los casos de chikungunya que se han presentado en el cantón Machala. La presencia de chikungunya estuvo a partir de la semana 15 con un caso, el pico se dio en la semana 25 y en las semanas 28 y 29 ocurrió un descenso paulatino, evidenciándose una transmisión propagada con presencia de pocos casos hasta la semana 52. 
Figura 2. Canal endémico de dengue año 2015, Machala.

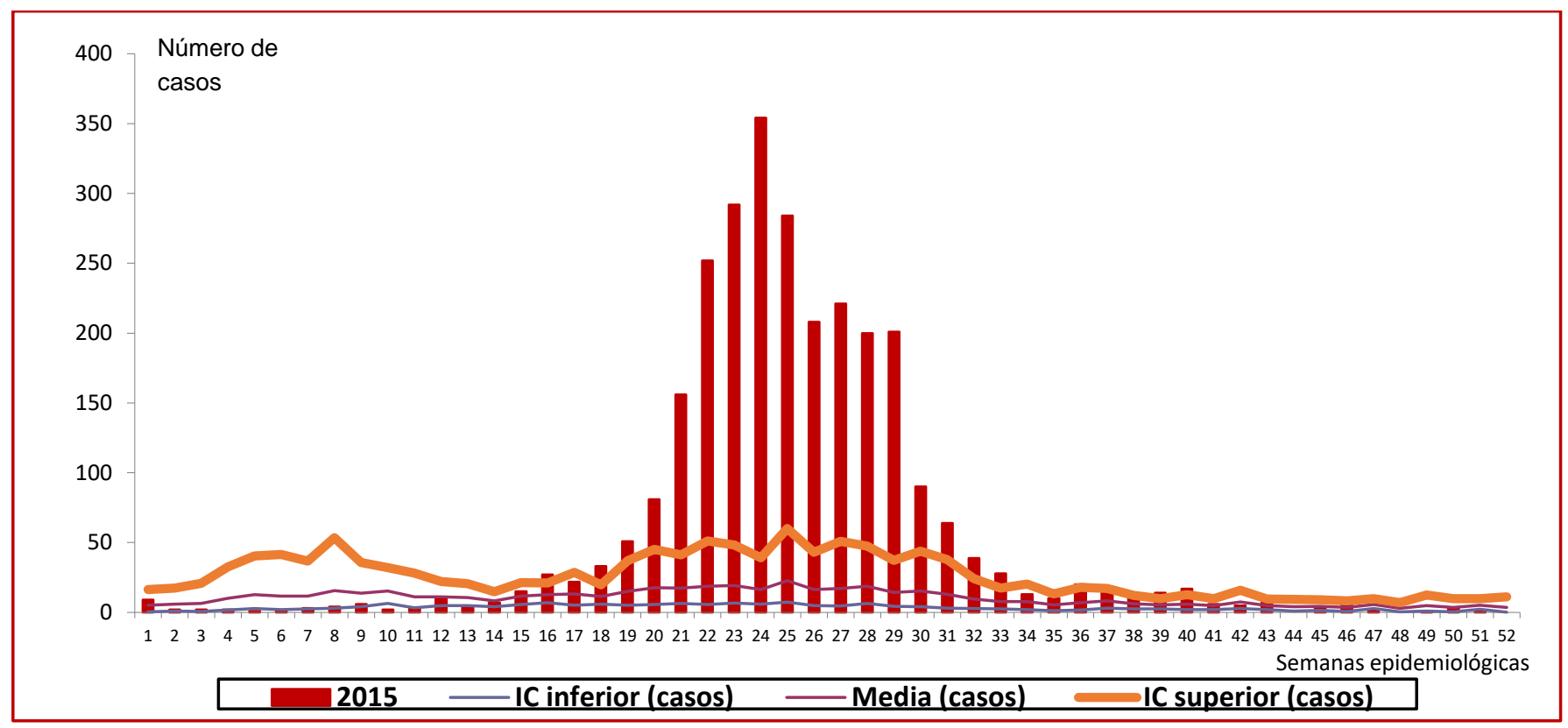

Fuente: Notificación del Sistema de Vigilancia Epidemiológica.

Tabla 1. Caracterización de casos de dengue según edad y sexo, 2015, Machala.

\begin{tabular}{|c|c|c|c|c|c|c|}
\hline \multirow{2}{*}{$\begin{array}{l}\text { Grupo de edad } \\
\text { por ciclos de vida }\end{array}$} & \multicolumn{2}{|c|}{ Masculino } & \multicolumn{2}{|c|}{ Femenino } & \multicolumn{2}{|c|}{ Total } \\
\hline & $\mathbf{N}$ & $\%$ & $\mathbf{N}$ & $\%$ & $\mathbf{N}$ & $\%$ \\
\hline$<1$ año & 7 & 0.2 & 10 & 0.4 & 17 & 0.6 \\
\hline 1 a 4 años & 76 & 2.7 & 52 & 1.8 & 128 & 4.6 \\
\hline 5 a 9 años & 141 & 5.0 & 121 & 4.3 & 262 & 9.3 \\
\hline 10 a 14 años & 222 & 7.9 & 162 & 5.8 & 384 & 13.7 \\
\hline 15 a 19 años & 140 & 5.0 & 154 & 5.5 & 294 & 10.5 \\
\hline 20 a 49 años & 463 & 16.5 & 794 & 28.2 & 1257 & 44.7 \\
\hline 50 a 64 años & 138 & 4.9 & 194 & 6.9 & 332 & 11.8 \\
\hline 65 años y más & 58 & 2.1 & 79 & 2.8 & 137 & 4.9 \\
\hline Total & 1245 & 44.3 & 1566 & 55.7 & 2811 & 100 \\
\hline
\end{tabular}

Fuente: Notificación del Sistema de Vigilancia Epidemiológica.

Figura 3. Curva Epidémica de Chikungunya por semanas, 2015, Machala.

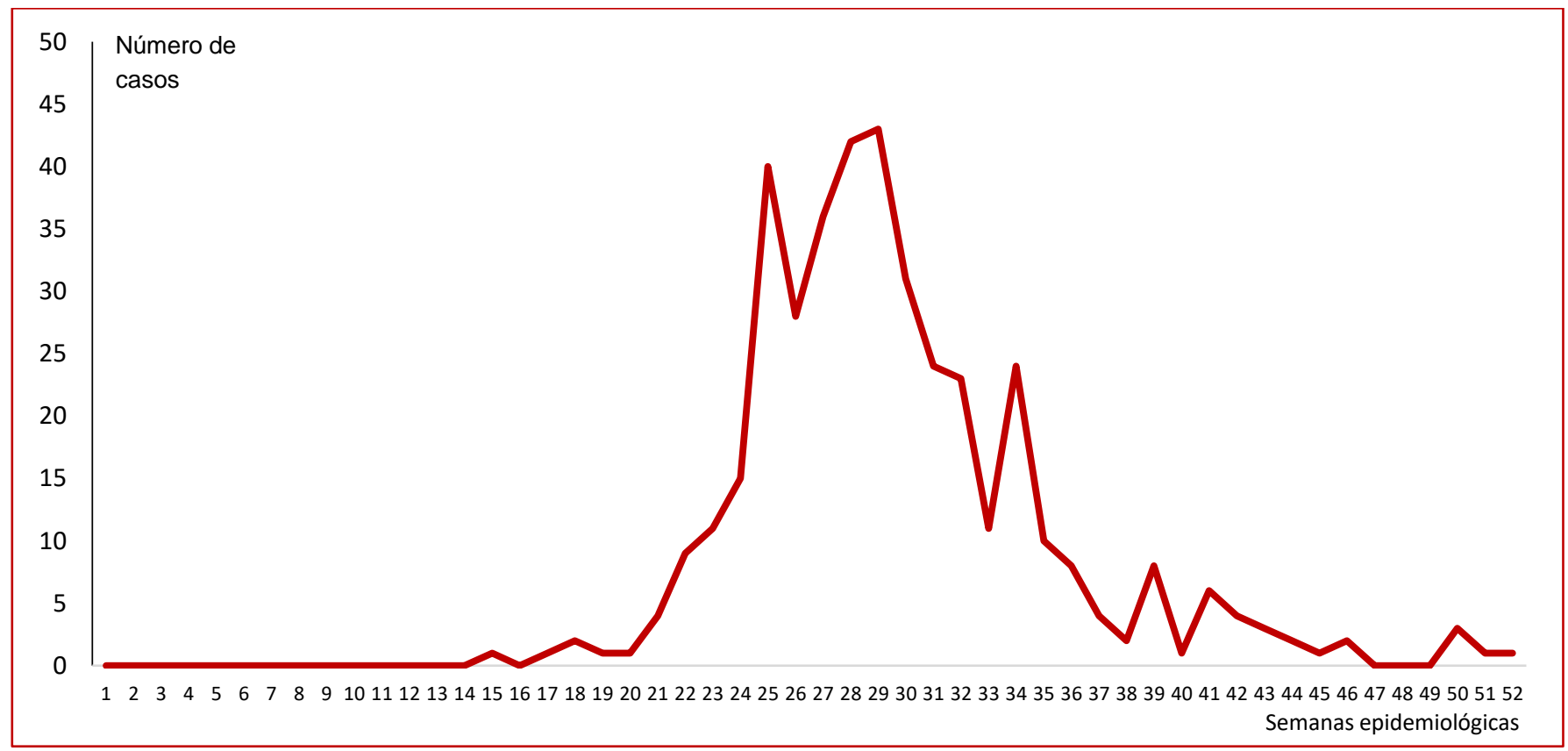


En el grupo de edades de 20 a 49 años se presentaron 208 casos (51.6\%), seguido del grupo de edad de 50 a 64 (16.6\%). El sexo más afectado fue el femenino con el $72.2 \%$, por lo que existió una razón de 26 mujeres frente a 10 varones. La presencia de Aedes aegypti en el interior de las casas podría aumentar el riesgo de trasmisión para el sexo femenino por la convivencia ser humano-vector (tabla 2).

En la figura 4 se observa que los casos de dengue superaron a los de chikungunya. Esta situación está en la función de la permanencia de los factores de riesgo como son la presencia del mosquito Aedes aegypti, el clima tropical, exposición de las personas al vector, sumándose en dengue la presencia de cuatro serotipos, la tendencia del dengue se mantenga dentro de las primeras causas de morbilidad. El hecho que marca el riesgo de gravedad es la introducción de un nuevo serotipo de dengue en la población sensibilizada con antecedente de haber adquirido dengue; mientras el chikungunya solo presenta un serotipo con una inmunidad duradera para ese serotipo, y por lo tanto el riesgo es menor a pesar de que coinciden los otros factores de riesgo.

Durante cuatro meses de trabajo de los visitadores, fueron inspeccionadas 116264 casas, con 5595 casas positivas (4.8\%), en tanto los depósitos identificados fueron 545769 , con 8022 depósitos positivos para Aedes aegypti, en los cuales se dio de un índice de depósitos positivos de $1.47 \%$. El índice de Breteau se encontró en $6.8 \%$, estando en condiciones de alerta el cantón Machala, se consideró el estándar de menos del $5 \%$ de este indicador.

Tabla 2. Distribución de casos de chikungunya según edad y sexo, 2015, Machala.

\begin{tabular}{|c|c|c|c|c|c|c|}
\hline \multirow{2}{*}{$\begin{array}{l}\text { Grupos de edad } \\
\text { por ciclos de vida }\end{array}$} & \multicolumn{2}{|c|}{ Masculino } & \multicolumn{2}{|c|}{ Femenino } & \multicolumn{2}{|c|}{ Total } \\
\hline & $\mathbf{N}$ & $\%$ & $\mathbf{N}$ & $\%$ & $\mathbf{N}$ & $\%$ \\
\hline$<1$ año & 10 & 2.5 & 12 & 3.0 & 22 & 5.5 \\
\hline 1 a 4 años & 3 & 0.7 & 7 & 1.7 & 10 & 2.5 \\
\hline 5 a 9 años & 10 & 2.5 & 4 & 1.0 & 14 & 3.5 \\
\hline 10 a 14 años & 9 & 2.2 & 22 & 5.5 & 31 & 7.7 \\
\hline 15 a 19 años & 9 & 2.2 & 18 & 4.5 & 27 & 6.7 \\
\hline 20 a 49 años & 51 & 12.7 & 157 & 39.0 & 208 & 51.6 \\
\hline 50 a 64 años & 11 & 2.7 & 56 & 13.9 & 67 & 16.6 \\
\hline 65 años y más & 9 & 2.2 & 15 & 3.7 & 24 & 6.0 \\
\hline Total & 112 & 27.8 & 291 & 72.2 & 403 & 100 \\
\hline
\end{tabular}

Fuente: Notificación del Sistema de Vigilancia Epidemiológica.

Figura 4. Comparación de Dengue y Chikungunya por semanas epidemiológicas, 2015, Machala.

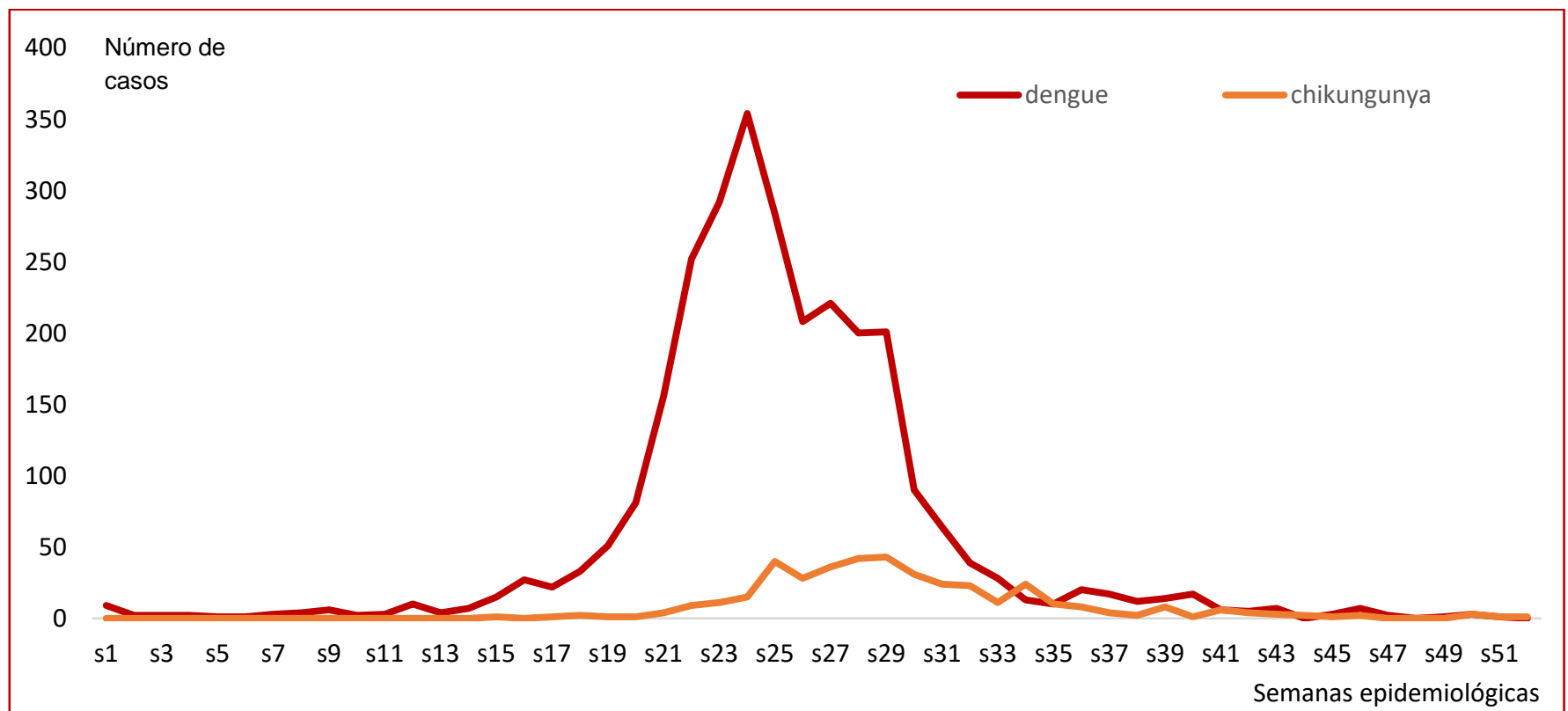

Fuente: Notificación del Sistema de Vigilancia Epidemiológica.

\section{Discusión}

La introducción del Chikungunya vino a poner en prueba a los equipos de salud, por las intervenciones que se implementaron, ya que el dengue en el cantón data del año 1989, cuando se vigiló de forma incipiente y actualmente es un evento que se vigila y controla con rigurosidad por las epidemias presentadas a nivel local. Situación dada en Latinoamérica de forma cíclica cada tres a cinco años ${ }^{1}$, por lo que las medidas de intervención deben ser oportunas y efectivas.
La presencia de los casos de chikungunya se dieron al mismo tiempo que los casos de dengue, en la estación invernal (enero a mayo). La transmisión estuvo en función de la curva como una enfermedad propagada de forma indirecta a través de la picadura del mosquito Aedes aegypti como epidemia, por estar fuera del canal endémico. Uno de los factores de riesgo estuvo en los estilos de vida de la población que se exponen a la hora de picada del vector, mayormente en la mañana y en la tarde, al grupo poblacional femenino, que se encuentra dentro de la vivienda. 
No se puede dejar de lado el factor ambiental, que denota claramente dos tipos de problemas que se presentan, como son el doméstico por ser los depósitos de tanques bajos el mayor problema de criaderos positivos, mientras la presencia de depósitos varios que denotan un problema de salubridad ambiental al ser tarrinas, tapas y botellas entre otras. La presencia de llantas en la comunidad es un problema ambiental como potenciales criaderos. El Aedes aegypti tiene un perfecto nicho ecológico para su reproducción aportando a la dinámica el cambio climático, movilización de las personas, acceso a servicios básicos y evolución viral ${ }^{1}$, situación que a nivel local repercute con las practicas que no son adecuadas para la prevención de estas enfermedades vectoriales.

El impacto de la presencia de chikungunya en Machala fue bajo, pues el número de casos presentados no fueron los que se esperaban, a pesar de la población susceptible existente por tratarse de la introducción de un nuevo virus. Las intervenciones vectoriales ejecutadas, están orientadas al fomento de estilos saludables de vida con la identificación y eliminación de depósitos de inservibles como potenciales criaderos que tienen las viviendas, en la prevención con la identificación y tratamiento de depósitos positivos para presencia de Aedes aegypti, el mismo que ha sido con larvicida ya sea biológico o químico de acuerdo a la necesidad poblacional.

\section{Conclusiones}

La presencia de dengue fue endémica durante todo el año exacerbándose los casos en la estación invernal y se incrementaron en el año 2015, afectó mayormente a personas entre 20 y 49 años de edad. El chikungunya afectó más a mujeres que a hombres. Entre las medidas implementadas para la prevención y control del dengue y chikungunya, estuvo la fumigación en localidades de riesgo, visitas domiciliarias realizadas y utilización del larvicida. Coincidieron los factores de riesgo para ambas enfermedades como la presencia del mismo vector transmisor, población expuesta al vector por las prácticas culturales y clima tropical. No obstante, la diferencia entre dengue y chikungunya radicó en los cuatros serotipos para dengue y uno para chikungunya, determinando el riesgo y la tendencia mayor para el caso del dengue.

\section{Conflicto de intereses}

Ninguno declarado por los autores.

\section{Agradecimientos}

Ninguno declarado por los autores.

\section{Referencias}

1. Murray NEA, Quam MB, Wilder-Smith A. Epidemiology of dengue: past, present and future prospects. Clinical Epidemiology [en línea]. 2013 [citado 25 de septiembre de 2017];5:299-309. Disponible en: http://www.ncbi.nlm.nih.gov/pmc/articles/PMC3753061/
2. Bhatt S, Gething PW, Brady OJ, Messina JP, Farlow AW, Moyes CL, et al. The global distribution and burden of dengue. Nature [en línea]. 7 de abril de 2013 [citado 5 de septiembre de 2017];496:504. Disponible en: http://dx.doi.org/10.1038/nature12060

3. Burke DS, Nisalak A, Johnson DE, Scott RM. A prospective study of dengue infections in Bangkok. The American journal of tropical medicine and hygiene [en línea]. 1988 [citado 5 de septiembre de 2017];38(1):172-80. Disponible

http://www.ajtmh.org/content/journals/10.4269/ajtmh.1988.38.172

4. Gustavo K. El dengue, un problema creciente de salud en las Américas. Revista Panamericana de Salud Pública. 2006;19(3):143145.

5. Diamond MS, Pierson TC. Molecular Insight into Dengue Virus Pathogenesis and Its Implications for Disease Control. Cell [en línea]. 30 de julio de 2015 [citado 25 de septiembre de 2017];162(3):488-92. Disponible

en http://www.sciencedirect.com/science/article/pii/S0092867415008429

6. Robinson LN, Tharakaraman K, Rowley KJ, Costa VV, Chan KR, Wong $\mathrm{YH}$, et al. Structure-Guided Design of an Anti-dengue Antibody Directed to a Non-immunodominant Epitope. Cell [en línea]. 30 de julio de 2015 [citado 5 de septiembre de 2017];162(3):493-504. Disponible en:

http://www.sciencedirect.com/science/article/pii/S0092867415008272

7. Taguwa S, Maringer K, Li X, Bernal-Rubio D, Rauch JN, Gestwicki JE, et al. Defining Hsp70 Subnetworks in Dengue Virus Replication Reveals Key Vulnerability in Flavivirus Infection. Cell [en línea]. 19 de noviembre de 2015 [citado 25 de septiembre de 2017];163(5):1108$23 . \quad$ Disponible en: http://www.sciencedirect.com/science/article/pii/S0092867415014026

8. McBride CS, Baier F, Omondi AB, Spitzer SA, Lutomiah J, Sang R, et al. Evolution of mosquito preference for humans linked to an odorant receptor. Nature [en línea]. 2014 [citado 25 de septiembre de 2017]:515(7526):222-7. Disponible en: https://www.nature.com/articles/nature13964

9. Schmid MA, Glasner DR, Shah S, Michlmayr D, Kramer LD, Harris E. Mosquito Saliva Increases Endothelial Permeability in the Skin, Immune Cell Migration, and Dengue Pathogenesis during AntibodyDependent Enhancement. PLOS Pathogens [en línea]. 16 de junio de 2016 [citado 25 de septiembre de 2017];12(6):e1005676. Disponible en: https://doi.org/10.1371/journal.ppat.1005676

10. Úsuga-Monroy C, Echeverri JJ, López-Herrera A. Asociación entre la infección por virus de la leucosis bovina y parámetros reproductivos en ganado Holstein-Colombiano. Actas Iberoamericanas de Conservación Animal [en línea]. 2015 [citado 25 de septiembre de 2017]:6(unknown):419-423 Disponible en: http://repositorio.unan.edu.ni/4442/1/96783.pdf

11. de Lamballerie X, Leroy E, Charrel RN, Ttsetsarkin K, Higgs S, Gould EA. Chikungunya virus adapts to tiger mosquito via evolutionary convergence: a sign of things to come? Virology Journal [en línea]. 2008 [citado 11 de septiembre de 2017];5(1):33. Disponible en: http://virologyj.biomedcentral.com/articles/10.1186/1743-422X-5-33

12. Orellano PW, Reynoso JI. Nuevo método para elaborar corredores endémicos. Rev Panam Salud Publica, Rev panam salud pública [en línea]. mayo de 2011 [citado 11 de septiembre de 2017];29:309-14. Disponible en: https://scielosp.org/pdf/rpsp/2011.v29n5/309-314

13. Shidrawi GR. Programa mundial de la OMS para la vigilancia de vectores resistentes a los plaguicidas [en línea] 1992 [citado 11 de septiembre de 2017]; Disponible en: http://iris.paho.org/xmlui/bitstream/handle/123456789/16440/v113n3p 223.pdf? sequence $=1$ \&isAllowed $=y$

14. Bhatt S, Gething PW, Brady OJ, Messina JP, Farlow AW, Moyes CL, et al. The global distribution and burden of dengue. Nature [en línea]. 7 de abril de 2013 [citado 11 de septiembre de 2017];496:504. Disponible en: http://dx.doi.org/10.1038/nature12060

15. Grard G, Caron M, Mombo IM, Nkoghe D, Mboui Ondo S, Jiolle D, et al. Zika Virus in Gabon (Central Africa) - 2007: A New Threat from Aedes albopictus? PLOS Neglected Tropical Diseases [en línea]. 6 de febrero de 2014 [citado 11 de septiembre de 2017];8(2):e2681. Disponible en: https://doi.org/10.1371/journal.pntd.0002681 